\title{
Thymidine kinase 1 expression in atypical ductal hyperplasia significantly differs from usual ductal hyperplasia and ductal carcinoma in situ: A useful tool in tumor therapy management
}

\author{
HONG GUAN $^{1}$, YANHUA SUN ${ }^{1}$, QIN ZAN ${ }^{1}$, MEIQUAN XU ${ }^{1}$, YUAN $\mathrm{LI}^{2}$, \\ JI ZHOU ${ }^{2}$, ELLEN HE ${ }^{2}$, STAFFAN ERIKSSON ${ }^{3}$, WEN WEN ${ }^{1}$ and SVEN SKOG ${ }^{2}$ \\ ${ }^{1}$ Department of Pathology, Shenzhen Second Hospital; ${ }^{2}$ Sino-Swed Molecular Bio-Medicine \\ Research Institute, Shenzhen, P.R. China; ${ }^{3}$ Department of Anatomy, Physiology and Biochemistry, \\ Swedish University of Agricultural Science, Biomedical Center, Uppsala, Sweden
}

Received March 26, 2009; Accepted July 10, 2009

DOI: $10.3892 / \mathrm{mmr} 00000193$

\begin{abstract}
Thymidine kinase 1 (TK1) is an enzyme involved in the synthesis of DNA precursors. In studies using immunohistochemistry, it was reported to be a more useful proliferation marker than Ki-67 and PCNA in breast, lung and colorectal carcinoma. In this study, we extend the work of prior breast carcinoma studies by investigating the expression of TK1 in 132 patients with usual ductal hyperplasia (UDH), atypical ductal hyperplasia (ADH), ductal carcinoma in situ (DCIS) and invasive ductal carcinoma (IDC). TK1 and Ki-67 expression were determined with monoclonal antibodies using the SP technique. The expression of TK1 was found to be significantly increased in the breast ductal carcinomas in the following manner: $\mathrm{UDH}<\mathrm{ADH}<\mathrm{DCIS}<\mathrm{IDC}$. TK1 expression was correlated with histological grade in DCIS and IDC patients and with pathological stage in IDC patients. The degree of TK1positive staining in the tumors of patients with ADH, DCIS and IDC was $80-90 \%$, while the corresponding value for UDH patients was less than 5\%. TK1 and Ki-67 expression showed very high correlation in the four tumor groups investigated. Since the expression of TK1 varied significantly between the breast ductal subgroups, we concluded that TK1 is a reliable proliferation marker for the determination of breast tumor proliferation, particularly of pre-cancerous lesions (ADH).
\end{abstract}

Correspondence to: Professor Sven Skog, Sino-Swed Molecular Bio-Medicine Research Institute, No. 2-304 Bio-Tech Industry Incubator, High-Tech Industrial Park, Gaoxin C. Ave. 1, 518057 Shenzhen, P.R. China

E-mail:svenisak@hotmail.com

Dr Hong Guan, Department of Pathology, Shenzhen Second Hospital, 3002 Shungang West Road, Shenzhen, P.R. China

E-mail:szgh999@sina.com

Key words: thymidine kinase 1, Ki-67, ductal breast cancer, usual ductal hyperplasia, atypical ductal hyperplasia, ductal carcinoma in situ, invasive ductal carcinoma, immunohistochemistry
This may enable timely treatment in the early stages of tumor development using minimal access surgery, thus avoiding extensive radical breast surgery and improving survival rates and the quality of life.

\section{Introduction}

The efficiency of breast cancer treatments has improved drastically over the last decades. For example, neo-adjuvant pre-operative chemotherapy, in combination with radiotherapy, endocrine and immunological treatments, results in a more effective therapy, reducing the risk of recurrence and improving survival rates and the quality of life (1-3). However, anti-cancer therapy is not yet highly effective, in particular in patients with later-stage breast cancer disease. Therefore, the development of methods suitable for the early detection of breast tumors is of high priority.

The detection of pre-cancerous lesions together with minimal access surgery is an effective cancer treatment strategy, and avoids extensive radical breast surgery. The use in health centers of regular breast cancer screening techniques, such as mammography and B-ultrasound, aids in the diagnosis of women with breast mass, while pathological examinations are used to identify benign tumors or pre-cancerous lesions. However, pathological examination does not determine the proliferation rate of breast tumors, and must therefore be combined with the use of proliferation markers.

In this study, we used regular breast cancer screening to identify patients with usual ductal hyperplasia (UDH) and atypical ductal hyperplasia (ADH), and investigated the expression of the proliferation markers thymidine kinase 1 (TK1) and $\mathrm{Ki}-67$ in these patients. TK1 and Ki-67 expression were found to differ between the breast tumor samples, being low in $\mathrm{UDH}$, significantly increased in $\mathrm{ADH}$, and further elevated in ductal carcinoma in situ (DCIS) and invasive ductal carcinoma (IDC). The pre-cancerous lesions also exhibited large individual variation in proliferation rate according to TK1 and Ki-67 expression. These results provide crucial information, and may serve as the basis for future treatment programs. 


\section{Materials and methods}

Patients and specimens. Breast cancer patients with UDH (benign), ADH (pre-malignant), DCIS (primary) and IDC were selected for the evaluation of TK1 expression. Patients with $\mathrm{UDH}(\mathrm{n}=25)$ and $\mathrm{ADH}(\mathrm{n}=22)$ were diagnosed during regular breast cancer screening using mammography and B-ultrasound at the Health Center of Shenzhen Second Hospital, Shenzhen, P.R. China, during 2007-2008. Specimens from women with breast mass were pathologically examined for the determination of UDH or ADH. Histopathologically normal breast ductal tissues $(n=20)$ were collected from the UDH patients far from the UDH tissue and used as controls. Patients with IDC $(n=60)$ and DCIS $(n=25)$ were recruited at the Pathology Department of Shenzhen Second Hospital during 2007-2008. The tumor grade and pathological stage of the IDC and DCIS specimens were determined according to the WHO tumor grading system and the AJCC Cancer Staging System (4), respectively, and were as follows: IDC grade $1, \mathrm{n}=12 ; 2, \mathrm{n}=34 ; 3, \mathrm{n}=14$. IDC stage I, $\mathrm{n}=23$; IIa, $\mathrm{n}=19$; IIb, $\mathrm{n}=16$; IIIa, $\mathrm{n}=2$. DCIS grade 1 , $\mathrm{n}=12 ; 2, \mathrm{n}=11 ; 3, \mathrm{n}=2$. All DCIS patients were of stage 0 . Premalignant ADH and flat epithelial atypia (FEA) were diagnosed according to the WHO recommendation (5). All patients gave their informed consent, and the study was performed in accordance with the Declaration of Helsinki of 1983.

Immunohistochemistry. Sections (4- $\mu \mathrm{m}$ thick) were prepared from formalin-fixed paraffin-embedded specimens by deparaffinization and rehydration. In order to unmask the TK1 activity in the tissue, the sections were incubated with Target Retrieval solution (Dako, Copenhagen, Denmark). Histochemical staining was carried out using the Dako EnVision ${ }^{\mathrm{TM}}$ System according to the manufacturer's instructions. In brief, sections were incubated for 5 min using $3 \% \mathrm{H}_{2} \mathrm{O}_{2}$ to block endogenous peroxidase activity. Non-specific binding sites were blocked by incubation with blocking buffer included in the EnVision kit. After incubation with the anti-TK1 monoclonal antibody (mAb) (800 X PBS dilution in $1 \mathrm{mg} / \mathrm{ml}$; SSTK Ltd., Shenzhen, P.R. China) or anti-Ki-67 mAb (40X PBS dilution in $1 \mu \mathrm{g} / \mathrm{ml}$, MIB-1, $50 \mathrm{mg}$ IgG1/l; Dako) for $2 \mathrm{~h}$ at room temperature, the sections were rinsed in PBS. EnVision conjugate was added, then the sections were incubated for $40 \mathrm{~min}$ at room temperature. Diaminobenzidine was used as a chromogen, and the slides were counterstained with haematoxylin. In order to evaluate the specificity of the $\mathrm{mAb}$ in the tumor tissues, normal tissues were also stained. No staining was found in normal tissues from nine out of ten patients. Immunostaining of TK1-positive and TK1-negative human lymphoma CEM cells was performed as described for the tumor tissues, except that the CEM cells were fixed in aceton.

Evaluation of immunohistochemistry. TK1-positive cells in each tissue section were counted among $\geq 1000$ cells in $\geq 10$ microscopic fields at a magnification of $x 200-400$, and expressed as the percentage of stained cells.

Production and characterization of anti-thymidine kinase 1 monoclonal antibody. mAb against TK1 was produced in mice as described elsewere (Gasparri $\mathrm{F}$ et al, Euro J Cell Biol, in press). Briefly, female Balb/c mice were immunized with a 31-mer peptide corresponding to the carboxyl-terminal end of TK1 at the Laboratory of Biochemistry and Molecular Biology, School of Life Science, Yunnan University, Kunming, P.R. China, for SSTK Ltd. Splenocytes were isolated and fused with the mouse myeloma cell line SP 2/0. Hybridomas were selected by screening the supernatants in ELISA plates coated with the 31-mer peptide. To characterize the specificity of the anti-TK1 antibody, cytochemical staining of CEM TK1positive and -negative cells (6) and native PAGE electrophoresis $(6,7)$ were performed as previously described.

The human lymphoma CEM cell lines, generously provided by B. Ullman (Oregon Health Science University, OR, USA) (8), were characterized as $\mathrm{TK} 1^{+}$wild-type $\left(\mathrm{TK} 1^{+} \mathrm{CEM}\right)$ or TK1-negative (TK1 - CEM). The latter were selected for bromodeoxyuridine resistance. Cells were cultured as previously described (6), lysed in lysis buffer [10 mM Tris, $137 \mathrm{mM}$ $\mathrm{NaCl}, 250 \mathrm{mM}$ sucrose, $1.5 \mathrm{mM} \mathrm{MgCl} 2,3.5 \mathrm{mM} \mathrm{NaF}, 2 \mathrm{mM}$ dithiothreitol (DTT), $1 \mathrm{mM}$ phenylmethylsulfonyl fluoride (PMSF), $0.05 \% \mathrm{NP}-40$ ] for $30 \mathrm{~min}$ at $4^{\circ} \mathrm{C}$, then centrifuged at $48,000 \mathrm{x} \mathrm{g}$ for $30 \mathrm{~min}$ at $4^{\circ} \mathrm{C}$. The supernatant was stored at $-70^{\circ} \mathrm{C}$ after the addition of $20 \%(\mathrm{v} / \mathrm{v})$ glycerol.

For native PAGE, $20 \mu \mathrm{g}$ of lysates from TK1+ CEM and TK1 ${ }^{-}$CEM cells were respectively added to an equal volume of non-denaturing sample buffer (125 mM Tris, $137 \mathrm{mM} \mathrm{NaCl}$, $20 \%$ glycerol, $\mathrm{pH} 8.8$ ) and then directly applied to a $4-10 \%$ native PAGE gradient gel $(6,7)$. Electrophoresis was performed according to the manufacturer's protocol (Bio-Rad, USA).

Statistical analysis. The mean values of TK1 were calculated using a mean and standard deviation program. The Student's t-test was used to calculate the statistical significance between groups of patients. Differences were considered significant at p-values $<0.05$.

\section{Results}

Characterization of anti-thymidine kinase 1 monoclonal antibody. The 31 peptide anti-TK1 mAb was characterized by immunohistochemical staining of TK1 ${ }^{-}$CEM and TK1 $1^{+}$ CEM cells (Fig. 1) and by native Western blot analysis (Fig. 2). While the $\mathrm{TK} 1^{+} \mathrm{CEM}$ cells were stained by the TK1 $\mathrm{mAb}$, no or very weak staining was found in the TK1- CEM cells (Fig. 1A and B). Native gel electrophoresis of cell extract from the $\mathrm{TK}^{+}$and TK1 $1^{-}$CEM cells revelead a strong band from the $\mathrm{TK}^{+}$cells, but no band from the TK1 $1^{-}$cells. Thus, this anti-TK1 mAb appears to be highly specific and useful in immunohistochemical experiments.

Characterization of histochemical staining of normal, benign and malignant breast tissues with anti-thymidine kinase 1 and Ki-67 monoclonal antibodies. The results of TK1 and $\mathrm{Ki}-67$ immunostaining are shown in Fig. 1. Cells from normal breast tissues showed negative or weak staining for TK1 and Ki-67 (Fig. 1C and D). In the UDH sections, few cells were stained. TK1 staining was apparent in the cytoplasm, while Ki-67 staining was located in the nucleus (Fig. 1E and F). The degree of staining in breast ductal carcinoma tissues increased from ADC patients (Fig. $1 \mathrm{G}$ and $\mathrm{H}$ ), through to DCIS patients (Fig. 1I and J) and on to patients with IDC (Fig. 1K and L). TK1 staining was located mainly in the cytoplasm, but was 


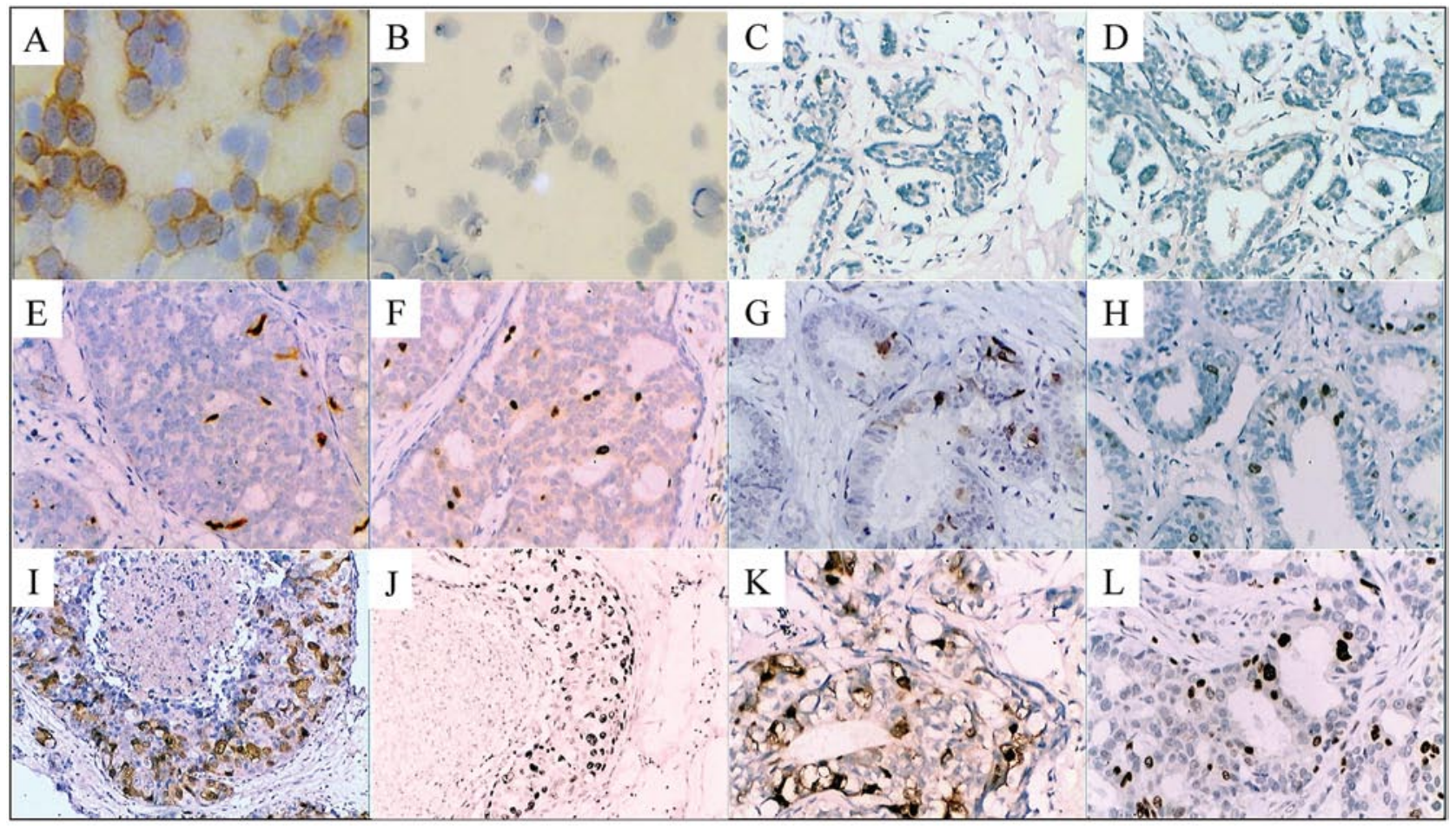

Figure 1. Immunohistochemical staining of $\mathrm{CEM}^{+}(\mathrm{A})$ and CEM- (B) cells, and of normal (C, TK1; D, Ki-67), benign UDH (E, TK1; F, Ki-67), pre-malignant ADH (G, TK1; H, Ki-67), primary DCIS (I, TK1; J, Ki-67) and IDC (K, TK1; L, Ki-67) breast tissues.

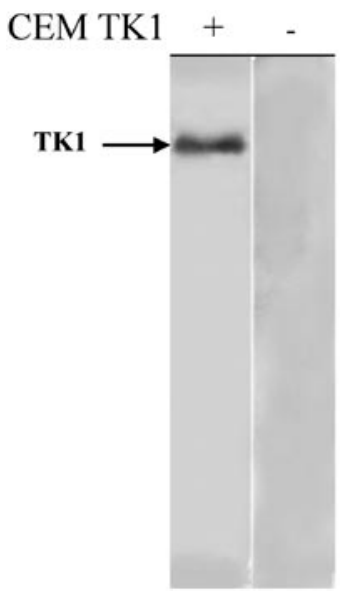

Figure 2. Native PAGE Western blot analysis with TK1 mAb of cell extracts from $\mathrm{CEM}^{+}$and $\mathrm{CEM}^{-}$cells.

also observed in the nucleus of some cells. As expected, Ki-67 staining was located in the nucleus.

Expression of thymidine kinase 1 and Ki-67 in normal tissues, benign tumors and ductal carcinomas. TK1 expression is presented on a per cell basis and gives the percentage of tumor cells stained in relation to total number of tumor cells. This makes it possible to determine differences in TK1 expression between various tumor groups. The results are shown in Fig. 3 and Table I. The expression of TK1 in the normal breast tissues was less than $1 \%$, while the mean TK1 expression of the UDH was significantly higher $(3.3 \pm 1.4 \%)$. The mean TK1 expression of the ADH tumors was $8.6 \pm 2.8 \%$, significantly

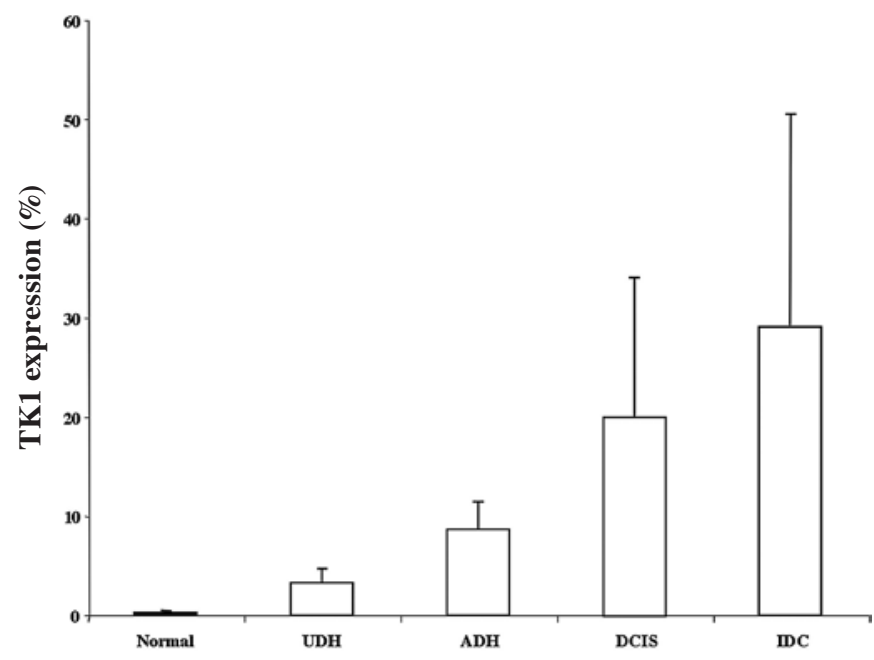

Figure 3. Expression of TK1 in benign UDH, pre-malignant ADH, primary DCIS and IDC breast tissues. TK1 expression is presented as the percentage of stained tumor cells. Values are the mean \pm SD.

different compared to TK1 expression in UDH ( $\mathrm{p}<0.001)$. No difference in TK1 expression was found between ADH and FEA types of pre-malignant tumors (data not shown). The mean TK1 expression increased significantly when patients with ADH developed into DCIS $(20 \pm 14 \%)$ and further to IDC $(29.1 \pm 21.4 \%)$. A level of TK1 expression of $>5 \%$ resulted in identification of $80-90 \%$ of TK1-positive patients with ADH, DCIS and IDC, while the UDH patients were positive in $10 \%$ of the cases. No significantly difference in the levels of expression of TK1 and Ki-67 was found among the four different groups of breast tumors in this study (Table II). 
Table I. Expression of TK1 and Ki-67 in normal breast and benign breast tissues (UDH), in pre-malignant (ADH), primary (DCIS) and invasive ductal (IDC) carcinoma of the breast.

\begin{tabular}{|c|c|c|c|c|c|c|}
\hline Type & Mean & SD & No. & Max & Min & P-value \\
\hline \multicolumn{7}{|c|}{ TK1 expression } \\
\hline Normal & 0.2 & 0.2 & 20 & 0.6 & 0.0 & \\
\hline UDH & 3.3 & 1.4 & 25 & 8.0 & 2.0 & $<0.001$ \\
\hline $\mathrm{ADH}$ & 8.6 & 2.8 & 22 & 13.5 & 3.0 & $<0.001$ \\
\hline DCIS & 20.0 & 14.0 & 25 & 55.8 & 4.2 & $<0.001$ \\
\hline IDC & 29.1 & 21.4 & 60 & 89.6 & 3.8 & 0.018 \\
\hline \multicolumn{7}{|c|}{ Ki-67 expression } \\
\hline Normal & 0.1 & 0.1 & 20 & 0.4 & 0.0 & \\
\hline UDH & 3.3 & 1.3 & 25 & 7.5 & 2.0 & $<0.001$ \\
\hline $\mathrm{ADH}$ & 8.6 & 2.8 & 22 & 13.1 & 3.2 & $<0.001$ \\
\hline DCIS & 20.0 & 14.0 & 25 & 55.3 & 4.0 & $<0.001$ \\
\hline IDC & 27.5 & 21.1 & 60 & 85.9 & 1.0 & 0.054 \\
\hline
\end{tabular}

Expression of TK1 and Ki-67 is presented as the percentage of stained tumor cells. Values are the mean \pm SD.

Table II. Expression of TK1 and Ki-67 in relation to the grade of primary breast ductal carcinoma in situ.

\begin{tabular}{|c|c|c|c|c|c|c|}
\hline Grade & Mean & $\mathrm{SD}$ & No. & $\operatorname{Max}$ & Min & P-value \\
\hline \multicolumn{7}{|c|}{ TK1 expression } \\
\hline 1 & 9.1 & 4.2 & 12 & 18.6 & 4.2 & \\
\hline 2 & 22.5 & 7.2 & 10 & 35.4 & 12.3 & $<0.001$ \\
\hline 3 & 49.2 & 8.1 & 3 & 55.8 & 40.2 & 0.014 \\
\hline \multicolumn{7}{|c|}{ Ki-67 expression } \\
\hline 1 & 9.2 & 4.3 & 12 & 18.4 & 4.0 & \\
\hline 2 & 23.0 & 7.6 & 10 & 36.1 & 40.5 & $<0.001$ \\
\hline 3 & 49.2 & 7.7 & 3 & 55.3 & 40.5 & 0.011 \\
\hline
\end{tabular}

Expression of TK1 and Ki-67 is presented as the percentage of stained tumor cells. Values are the mean \pm SD.

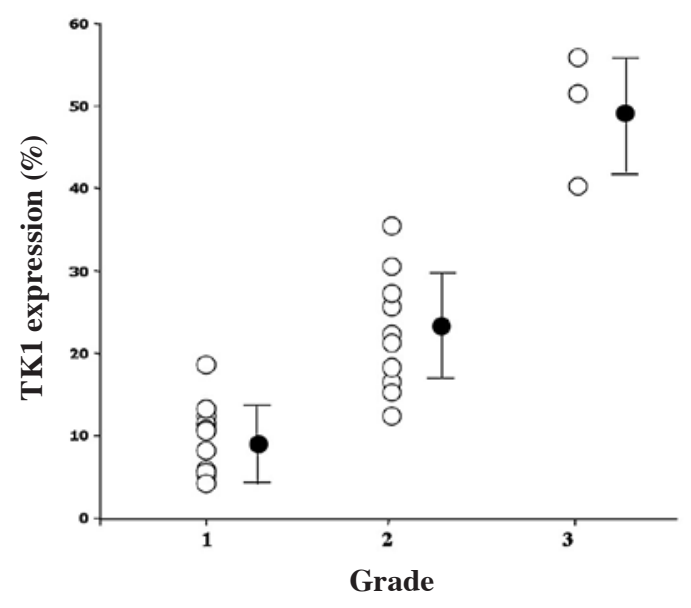

Figure 4. Expression of TK1 in relation to grade of primary breast ductal carcinoma in situ. TK1 expression is presented as the percentage of stained tumor cells. Values are the mean \pm SD.
Expression of thymidine kinase 1 and $\mathrm{Ki}-67$ in relation to tumor grade and stage of DCIS and IDC patients. There was a significant increase in TK1 expression from grade 1 to grade 3 in the DCIS patients (Fig. 4, Table II), as well as in the IDC patients (Fig. 5, Tables III and IV). Additionally, in the IDC patients, a correlation was observed between stage and TK1 expression, although the overlap between stages was extensive (Fig. 5, Tables III and IV). However, there was a significant increase in the mean expression of TK1 from stage I to stage IIa $(p=0.029)$ and from stage I to stages IIb and IIIa $(p<0.001)$, but not between stages IIa and IIb $(\mathrm{p}=0.196)$ (Tables III and IV).

Expression of TK1 was significantly higher in tumors $>3 \mathrm{~cm}$ compared to tumors $<3 \mathrm{~cm}$ in size (data not shown).

The correlation between TK1 and Ki-67 expression in the subgroups in terms of grade and stage was very high ( $\mathrm{r}=0.97$ 0.99), indicating that, in this case, neither marker was capable of distinguishing different sub-populations of proliferating cells. 
Table III. Expression of TK1 in relation to the grade and the stage of invasive breast ductal carcinoma.

\begin{tabular}{|c|c|c|c|c|c|c|}
\hline & Mean & $\mathrm{SD}$ & No. & Max & Min & P-value \\
\hline \multicolumn{7}{|c|}{ Grade } \\
\hline 1 & 6.8 & 3.4 & 12 & 15.0 & 3.8 & \\
\hline 2 & 25.1 & 11.4 & 34 & 54.6 & 8.8 & $<0.001$ \\
\hline 3 & 55.7 & 22.7 & 14 & 89.6 & 4.2 & $<0.001$ \\
\hline \multicolumn{7}{|l|}{ Stage } \\
\hline I & 17.0 & 13.4 & 22 & 45.3 & 3.8 & \\
\hline IIa & 29.3 & 19.0 & 20 & 78.2 & 4.7 & 0.029 \\
\hline $\mathrm{IIb}$ & 40.3 & 24.4 & 16 & 89.6 & 12.2 & 0.196 \\
\hline IIIa & 61.7 & 23.4 & 2 & 78.2 & 45.1 & 0.403 \\
\hline
\end{tabular}

Expression of TK1 is presented as the percentage of stained tumor cells. Values are the mean \pm SD.

Table IV. Expression of Ki-67 in relation to the grade and the stage of invasive breast ductal carcinoma.

\begin{tabular}{|c|c|c|c|c|c|c|}
\hline & Mean & $\mathrm{SD}$ & No. & Max & Min & P-value \\
\hline \multicolumn{7}{|c|}{ Grade } \\
\hline 1 & 5.5 & 3.2 & 12 & 14.1 & 1.0 & \\
\hline 2 & 23.7 & 11.1 & 34 & 50.8 & 7.5 & $<0.001$ \\
\hline 3 & 53.8 & 22.2 & 14 & 85.9 & 4.5 & $<0.001$ \\
\hline \multicolumn{7}{|l|}{ Stage } \\
\hline I & 15.7 & 13.2 & 23 & 43.6 & 1.0 & \\
\hline IIa & 28.0 & 18.6 & 20 & 75.6 & 4.2 & 0.027 \\
\hline IIb & 38.3 & 24.1 & 16 & 85.9 & 10.5 & 0.224 \\
\hline IIIa & 59.4 & 24.2 & 2 & 76.5 & 42.3 & 0.419 \\
\hline
\end{tabular}

Expression of Ki-67 is presented as the percentage of stained tumor cells. Values are the mean $\pm \mathrm{SD}$.

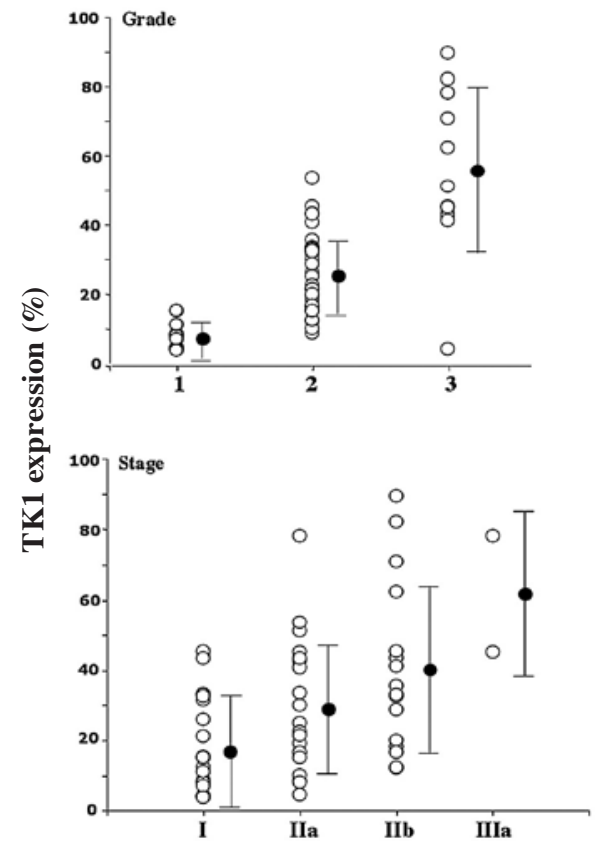

Figure 5. Expression of TK1 in relation to grade and stage of invasive breast ductal carcinoma. The expression of TK1 is presented as the percentage of stained tumor cells. Values are the mean $\pm \mathrm{SD}$.
Age distribution. The mean ages of the ADH, DCIS and IDC patients were $40.6 \pm 8.1,45.3 \pm 5.6$ and $50.3 \pm 13$ years, respectively (Table V). Differences between the ages were significantly different. The mean age of the UDH patients was $43.5 \pm 7.7$ years.

\section{Discussion}

Breast cancer is the second most common malignant tumor in Chinese females, and its incidence is steadily rising. The etiology of breast cancer is complex and is likely to involve the actions of genes at multiple levels along the multistage process of carcinogenesis. Different cells in normal and malignant breast tissues have different gene expression, making a complete characterization of the gene expression patterns of these cells by cDNA-array or proteomics techniques difficult $(9,10)$. To date, few tumor markers for breast cancer are in use $(11,12)$ that meet the criteria set by the American Society of Clinical Oncology (ASCO) (11). Steroid hormone receptors (estrogen and progesterone) are among the recommended markers, providing prognostic information and aid in planning breast cancer treatment strategy. However, the number of patients with estrogen-receptor (ER)-positive primary tumors is dependent on age, with $\sim 50 \%$ of pre-menopausal patients testing 
Table V. Mean ages of patients with benign (UDH) and pre-malignant (ADH) tumors, primary carcinoma in situ (DCIS) and invasive ductal carcinoma (IDC).

\begin{tabular}{lccccccc}
\hline Type & Mean & SD & Median & No. & Max & Min & P-value \\
\hline UDH & 43.5 & 7.7 & 43.0 & 25 & 58 & 23 & \\
ADH & 40.6 & 8.1 & 42.0 & 22 & 50 & 21 & 0.214 \\
DCIS & 45.3 & 5.6 & 45.0 & 25 & 62 & 33 & 0.031 \\
IDC & 50.3 & 13.0 & 49.5 & 60 & 83 & 29 & 0.016 \\
\hline
\end{tabular}

Values are the mean $\pm \mathrm{SD}$.

positive compared to $75-85 \%$ of menopausal patients. HER-2/ neu (c-erbB-2), a growth factor receptor overexpressed in $\sim 25-30 \%$ of the human breast cancer patients (12), is also recommended for use in the diagnosis and prognosis of breast cancer (13). PCNA and Ki-67 are commonly used markers related to cell proliferation that are applied for prognostic purposes. These markers are positive in $\sim 40-60 \%$ of tumor samples (14). However, as yet no studies have conclusively shown a relationship between $\mathrm{Ki}-67$ and the S-phase of the cell cycle (15). Moreover, PCNA shows some false immunoreactivity in G0 cells (16).

In clinical practice, the proliferating markers PCNA and $\mathrm{Ki}-67$ are still in use for patients with breast cancer, in particular Ki-67 (17). When the immunohistochemical staining of PCNA and $\mathrm{Ki}-67$ was compared to in vivo bromodeoxyuridine (BrdU) labeling, the labeling indexes (LIs) of these markers were ranked in the order $\mathrm{PCNA}<\mathrm{Ki}-67<\mathrm{BrdU}$, indicating that the PCNA and Ki-67 LIs did not match in vivo BrdU labeling (18). The BrdU LI reflects the synthesis rate of DNA. In pregnancy/ lactation-associated breast cancer patients, no significant difference was found between the overall survival of breast carcinoma patients vs. the control group, although significant differences in the expression of ER/progesterone receptor, epidermal growth factor receptor and PCNA (13) were found. HER-2/ neu and PCNA expression is clearly important from a biological standpoint, but they have not been recognized as independent molecular markers. In a recent study of breast cancer response to neo-adjuvant chemotherapy, Bcl-2, ER, p53, HER-2/neu and Ki-67 were used as predictive markers. ER negativity and a high proliferation index were associated with improved response, while HER-2/neu status did not predict response (19). Thus, there is a need for new prognostic markers that can provide direct benefits during the planning of anticancer therapy and the follow-up of patients with breast cancer.

TK1 is a cytosolic enzyme responsible for the phosphorylation of the DNA precursor thymidine. It is only expressed in S-phase/G2 cells, and the intracellular activity and concentration of this protein are known to be closely correlated with the cell proliferation of cultured cell lines and tissues, both normal and malignant (20-25).

The expression of TK1 is related to the cell cycle and is regulated in a complex manner; i.e., TK1 is activated during G1/S, peaks in the S-phase and is inactivated in G2/M. The carboxyl-terminal 40 residues of TK1 are essential for the cell cycle regulation of TK1 activity (20). Deletion of these residues completely abolishes cell cycle-dependent variations in activity. Therefore, we chose a peptide from this region and developed IgY and IgG anti-TK1 polyclonal antibodies $(7,25)$. In this study, IgG anti-TK1 mAb was used. The results indicated that the TK1 mAb is highly specific for the TK1 peptide and reacts with the native intact TK1 protein in both biochemical and cell biological analytic procedures.

The expression of TK activity has been studied in human breast cancer cell lines (26), in the cytosol of human primary breast tumors (27-29) and in the serum of human breast cancer patients (30-32). TK activity in the cytosol of breast tumors is the only factor negatively correlated with overall survival (30), and the only factor predictive of disease-free and metastasisfree survival in node-negative patients (30). However, only two studies have been conducted using sections of ductal breast carcinoma $(6,33)$ with TK1 as a proliferation marker. In the first of these, it was demonstrated using polyclonal TK1 antibodies that the number of patients with tumors positively stained for TK1 was significant higher (79\%) than the number of those positively stained for PCNA (65\%) (33). TK1 expression was also correlated with higher stage and grade, while PCNA was not (35). In the second study on ductal breast carcinoma using two types of TK1 mAbs, the number of patients with tumors positively stained fo TK1 was found to be $57 \%$, while the corresponding value for Ki-67 was $41 \%$ (6). The difference was only significant in patients with grade 2 tumors. However, from an anti-tumor therapy point of view, the identification of grade 2 patients is important.

In the present immunohistochemistry study with various types of ductal breast carcinoma (ADH, DCIS and IDC), we confirmed earlier results regarding TK1 and breast malignancy, showing TK1 expression to be correlated with stage and grade. Furthermore, mean TK1 expression was significantly higher in grade 2 DCIS and IDC tumors compared to grade 1 tumors, which demonstrates the applicability of TK1 as a prognostic factor in breast ductual carcinoma. This provides novel information regarding TK1 expression in breast malignancies: TK1 expression differs significantly between the various subgroups of ductal breast carcinoma patients. Furthermore, TK1 and Ki-67 expression measured by immunohistochemistry were surprisingly similar in this group of patients, suggesting that the determination of TK1 expression may aid in the planning of individual and improved anti-cancer therapy, particularly in patients with ADH and DCIS. For example, ADH and DCIS patients with high TK1 expression should likely be treated more aggressively than patients with low or no TK1 expression, and patients with ADH may qualify for minimal access surgery, 
avoiding extensive radical breast surgery. The use of a combination of mammography, B-ultrasound and proliferation markers such as TK1 or Ki-67 may help identify patients with early-stage disease, i.e., ADH/DCIS, providing a more accurate prognosis. In a recent study on DCIS based on a multivariate analysis of frequency of necrosis, survivin, Bax, Bls-2, Ki-67 and apoptotic index (34), it was demonstrated that DCIS tumors with micro-invasion were characterized by a slightly elevated cell proliferation capacity. This shows the importance of determining the proliferation rate of tumors in DCIS patients for the prognosis and treatment of the disease. TK1 expression appears to be suitable for this purpose.

The accuracy of the present study is shown by the patient age distribution in the various tumor subgroups. The mean ages of the ADH, DCIS and IDC patients were 40.6 $\pm 8.1,45.3 \pm 5.6$ and $50.3 \pm 13$ years, respectively, which are significantly different from each other and in accordance with the age distributions of breast cancer patients. For example, the Cancer Committee of the College of American Pathologists has assigned ADH patients a 4-5 times increased risk of subsequently developing IDC within 8.3 years (5).

In summary, our results indicate that TK1 expression as a tumor proliferating marker could be as useful as Ki-67 in determining the best course of tumor therapy for the various subgroups of breast ductal carcinoma. This is of particular interest for patients with pre-cancerous lesions, who may receive minimal access surgery instead of extensive radical breast surgery, improving survival rates and quality of the life.

\section{Acknowledgements}

This study was made possible by grants from the Cancer Society of Stockholm, the faculty of the Karolinska Institute, Stockholm, and the Clinical Research Center, Karolinska University Hospital, and by a grant to S.E. from the Swedish Research Council, Uppsala, Sweden. The anti-TK1 antibody was supplied by SSTK Ltd., Shenzhen, P.R. China.

\section{References}

1. Glück S, McKenna EF Jr and Royce M: XeNA: capecitabine plus docetaxel, with or without trastuzumab, as preoperative therapy for early breast cancer. Int J Med Sci 5: 341-346, 2008.

2. Torris R, Bagnardi V, Cardillo A, et al: Preoperative bevacizumab combined with letrozole and chemotherapy in locally advanced ER- and/or PgR-positive breast cancer: clinical and biological activity. Br J Cancer 18: 1564-1571, 2008.

3. Prati R, Minami CA, Gornbein JA, et al: Accuracy of clinical evaluation of locally advanced breast cancer in patients receiving neoadjuvant chemotherapy. Cancer 115: 1194-1202, 2009.

4. Fleming ID, Cooper JS, Henson DE, et al (eds): Breast. In: AJCC Cancer Staging Manual. 5th edition. Lippincott-Raven Publishers, Philadelphia, pp171-211, 1995.

5. Tavassoli FA and Devilee P: WHO Organization Classification of Tumors, Pathology and Genetics, Tumors of the Breast and Female Genital Organs. World Health Organization, p16, 2002.

6. He E, Mao Y, Wu J, et al: Cytosolic thymidine kinase is a specific histopathologic tumor marker for breast carcinomas. Int J Oncol 25: 945-953, 2004.

7. Wu C, Yang R, Zhou J, et al: Production and characterisation of a novel chicken IgY antibody raised against $\mathrm{C}$-terminal peptide from human thymidine kinase 1. J Immunol Methods 277: 157-169, 2003.

8. Törnevik Y, Ullman B, Balzarini $\mathrm{J}$, et al: Cytotoxicity of 3'-azido-3'-deoxythymidine correlates with 3'-azidothymidine5'-monophosphate (AZTMP) levels, whereas anti-human immunodeficiency virus (HIV) activity correlates with 3'-azidothymidine 5'-triphosphate (AZTTP) levels in cultured CEM T-lymphoblastoid cells. Biochem Pharmacol 49: 829-837, 1995.
9. Perou CM, Sørlie T, Eisen MB, et al: Molecular portraits of human breast tumors. Nature 406: 747-752, 2000.

10. Ahr A, Holtrich U, Solbach C, et al: Molecular classification of breast cancer patients by gene expression profiling. J Pathol 195: 312-320, 2001.

11. ASCO: Recommendations of the American Society of Clinical Oncology. A patient's guide: understanding tumor markers for breast and colorectal cancers. American Society of Clinical Oncology, pp1-7, 2002.

12. Beresford MJ, Wilson GD and Makris A: Measuring proliferation in breast cancer: practicalities and applications. Breast Cancer Res 8: 216, 2006.

13. Pegram M and Slamon D: Biological relation for Her/neu (c- erbB2) as a target for monoclonal antibody therapy. Semin Oncol 9: 13-19, 2000.

14. Aziz S, Pervez S, Khan S, et al: Case control study of novel prognostic markers and diseases outcome pregnancy/lactationassociated breast carcinoma. Pathol Res Pract 199: 15-21, 2003.

15. Scholzen T and Gerdes J: The Ki-67 protein: from the known and the unknown. J Cell Physiol 182: 311-322, 2000.

16. Eissa S: Tumor Markers. Chapman \& Hall Medical, New York, pp94-102; pp134-137, 1998.

17. Beenken SW and Bland KI: Biomarker for breast cancer. Minerva Chir 57: 437-448, 2002.

18. Zacchetti A, van Garderen E, Teske E, et al: Validation of the use of proliferation markers in canine neoplastic and non-neoplastic tissues: comparison of Ki-67 and proliferating cell nuclear antigen (PCNA) expression versus in vivo bromodeoxyuridine labeling by immunohistochemistry. APMIS 111: 430-438, 2003.

19. Faneyte IF, Schrama JG, Peterse JL, et al: Breast cancer response to neoadjuvant chemotherapy: predictive markers and relation with outcome. Br J Cancer. 88: 406-412, 2003.

20. Sherley JL and Kelly TJ: Regulation of human thymidine kinase during the cell cycle. J Biol Chem 26: 8350-8358, 1988.

21. Sherley JL and Kelly TJ: Human cytosolic thymidine kinase. Purification and physical characterization of the enzyme from HeLa cells. J Biol Chem 263: 375-382, 1988.

22. He Q, Skog S and Tribukait B: Cell cycle related studies on thymidine kinase and its isoenzymes in Ehrlich ascites tumors. Cell Prolif 24: 3-14, 1991.

23. Kauffman MG and Kelly TJ: Cell cycle regulation of thymidine kinase: residues near the carboxyl terminus are essential for the specific degradation of the enzyme at mitosis. Mol Cell Biol 11: 2538-2546, 1991.

24. Hengstschläger M, Knöfler M, Müllner EW, et al: Different regulation of thymidine kinase during the cell cycle of normal versus DNA tumor virus-transformed cells. J Biol Chem 19: 13836-13842, 1994.

25. He Q, Wang N, Skog S, et al: Characterization of a peptide antibody against a $\mathrm{C}$-terminal part of human and mouse cytosolic thymidine kinase, which is a marker for cell proliferation. Eur J Cell Biol 70: 117-124, 1996.

26. Schwartz GF, Schwarting R, Rabindranauth $\mathrm{P}$, et al: Clinical application of serum and tissue markers in malignant disease: breast cancer as the paradigm. Clin Chem 39: 2401-2412, 1993.

29. Broet P, Romain S, Daver A, et al: Thymidine kinase as a proliferative marker: clinical relevance in 1,692 primary breast cancer patients. J Clin Oncol 19: 2778-2787, 2000.

28. Romain S, Christensen IJ, Chinot O, et al: Prognostic value of cytosolic thymidine kinase activity as marker of proliferation in breast cancer. Int J Cancer 61: 7-12, 1995.

29. Romain S, Martin PM and Klijin JG: DNA-synthesis enzyme activity: a biological tool useful for predicting anti-metabolic drug sensitivity in breast cancer. Int J Cancer 74: 156-161, 1997.

30. Spyratos F, Martin PM, Hacene K, et al: Multiparametric prognostic evaluation of biological factors in primary breast cancer. $\mathbf{J}$ Natl Cancer Inst 84: 1266-1272, 1992.

31. He Q, Zou L, Zhang P, et al: The clinical significance of thymidine kinase 1 measurement in serum of breast cancer patients using anti-TK1 antibody. Int J Biol Marker 15: 139-146, 2000.

32. He Q, Fornander T, Johansson H, et al: Thymidine kinase 1 in serum predicts increased risk of distant or loco-regional recurrence following surgery in patients with early breast cancer. Anticancer Res 26: 4753-4759, 2006.

33. Mao YR, Wu JP, Wang N, et al: Comparison study: immunohistochemical detection of cytosolic thymidine kinase and proliferating cell nuclear antigen in breast cancer. Cancer Invest 20: 922-931, 2002.

34. Okumura Y, Yamamoto Y, Zhang Z, et al: Identification of biomarkers in ductal carcinoma in situ of breast with microinvasion. BMC Cancer 6: 287, 2008. 